\title{
Article \\ Synthesis and Fluorescent Properties of Novel Mono- and Di-Substituted 1,8-Naphthalimide Derivatives at the $C-4$ Position
}

\author{
Ying Fu, Xiao-Xiao Pang, Kui Wang, Zhi-Qiang Wang, Guan-Yu Li, Fei Ye * \\ Department of Applied Chemistry, College of Science, Northeast Agricultural University, \\ Harbin 150030, China; fuying@neau.edu.cn (Y.F.); pangxiaoxiaoyk@163.com (X.-X.P.); \\ abcwangkui@163.com (K.W.); wzq19910101@163.com (Z.-Q.W.); liguanyuat@163.com (G.-Y.L.) \\ *Corresponding Author: Fei Ye, Email address: yefei@neau.edu.cn; Tel.: +86-451-55191507
}

\begin{abstract}
A series of novel $N$-n-butyl-1,8-naphthalimide derivatives were synthesized via a three-step reaction involving nucleophilic substitution and acylation. All of the compounds were characterized by IR, ${ }^{1} \mathrm{H}$ NMR, ${ }^{13} \mathrm{C}$ NMR, MS, and elemental analysis, and the crystal structure of $N$-n-butyl-4-[N', $N^{\prime}$-bis(2',4'-dichlorobenzoyl)ethylamino]-1,8-naphthalimide was determined. The $\pi-\pi$ stacking interactions and hydrogen bonds between the two molecular core planes (naphthalimide ring) and the van der Waals forces between the flexible n-butyl groups resulted in a 3D long-chain structure. The UV-vis and fluorescence properties of the title compounds were investigated. The results indicated that the monosubstituted 1,8-naphthalimide derivatives bearing an electron-donating group on the benzene ring or a structure with a larger conjugative effect exhibited enhanced fluorescence properties.
\end{abstract}

Keywords: $N$-n-Butyl-4-( $N^{\prime}, N^{\prime}$-dihydroxyethylamino)-1,8-naphthalimide; mono-substituted; di-substituted; synthesis, crystal structure; fluorescence

\section{Introduction}

The development of fluorescent sensors for the selective detection of chemically and biologically significant metal ions has attracted attention worldwide [1-5]. The naphthalene moiety is an ideal fluorophore, and its derivatives have been widely applied as fluorescent dyes, metal sensors, optical sensors and organic light-emitting materials because of their high absorption coefficients, high fluorescence and quantum yields, large Stokes shifts, good photostability and easy modification [6-9]. In recent years, naphthalimide-based probes have been developed to detect $\mathrm{H}^{+}, \mathrm{Hg}^{2+}, \mathrm{Zn}^{2+}, \mathrm{Cu}^{2+}, \mathrm{Ag}^{+}, \mathrm{Cd}^{2+}, \mathrm{Pd}^{2+}, \mathrm{Cr}^{3+}, \mathrm{Al}^{3+}, \mathrm{Fe}^{3+}$, and $\mathrm{F}^{-}$via chromogenic and fluorogenic analyses [10-16]. Therefore, the naphthalimide derivatives are potential carriers that could be used in the preparation of new optical chemosensors. Although extensive studies have focused on 1,8-naphthalimide derivatives with an $O$ - [17,18], $N$ - $[19,20], S$-substituted group [21] or five-member heterocycles [22,23] at the C-4 position, extending the study of these materials, especially regarding introducing different electron-donating groups, could still produce useful results. Indeed, few studies focusing on fluorescent 1,8-naphthalimide derivatives containing a mono- or di-substituted ester at the C-4 position have been reported. Therefore, in this study, we designed and synthesized a series of $N$-n-butyl-1,8-naphthalimide fluorescent sensors with a diethylamino link to the $C-4$ position. The mono- and di-substituted esters were obtained simultaneously. In addition, a single crystal was prepared, and its spatial configuration was characterized with regard to its complexation with metal ions. The effects of protons and metal ions on the UV-vis absorption and fluorescence spectra of these sensors were also investigated. 


\section{Results and discussion}

\subsection{Synthesis and characterization}

As shown in Scheme 1, the mono- and di-substituted 1,8-naphthalimide derivatives were synthesized via three steps. Although many efforts have been made to prepare 1,8-naphthalimide derivatives with different substitutions at position 4 [1,10,11,27-30], this manuscript reports the first attempt to simultaneously design and synthesize mono- and di-substituted 1,8-naphthalimide derivatives at position 4 . N-n-Butyl-4-bromo-1,8-naphthalimide was prepared by the convenient substitution of 4-bromo-1,8-naphthlic anhydride with n-butylamine. The intermediate BNI was obtained through nucleophilic substitution with diethanolamine. The mono- and di-substituted products were obtained simultaneously with mild yields via an acylation reaction (Scheme 1 and Table 1).

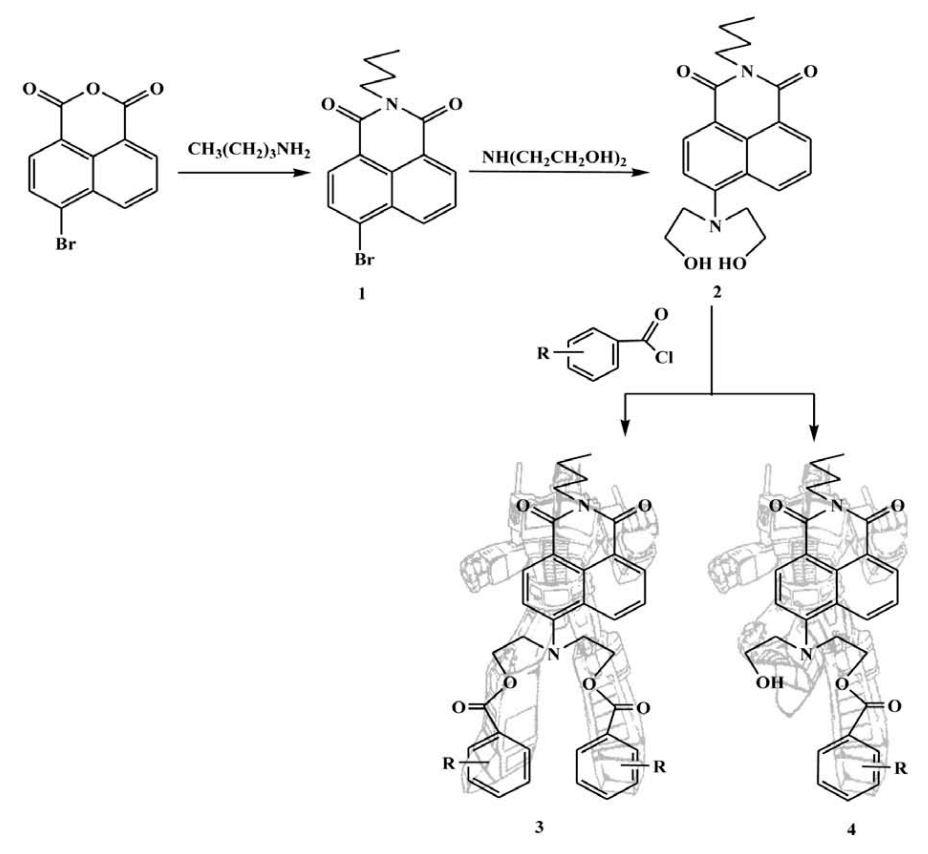

Scheme 1. Synthetic route for the production of compounds 3 and 4

Table 1. Synthesis of mono- and di-substituted 1,8-naphthalimide derivatives (a-f).

\begin{tabular}{|c|c|c|c|c|c|}
\hline Compound & Reactant & Yield of 3 & M.p. of $3^{a}$ & Yield of 4 & M.p. of $4^{\text {a }}$ \\
\hline a & & 46.0 & $148-150$ & 61.4 & $158-159$ \\
\hline$b$ & & 48.2 & 94-95 & 57.6 & $179-180$ \\
\hline c & & 30.4 & 104-105 & 61.2 & $168-169$ \\
\hline d & & 33.7 & $88-90$ & 60.5 & $167-168$ \\
\hline e & & 20.8 & $100-101$ & 50.4 & $161-162$ \\
\hline
\end{tabular}




\begin{tabular}{llllll}
\hline $\mathrm{Cl}$ & $\mathrm{O}$ & $67-88$ & 62.3 & $178-179$ \\
\hline
\end{tabular}

a All of the products were characterized through m.p., IR, ${ }^{1} \mathrm{H}$ NMR, ${ }^{13} \mathrm{C} N M R$, MS, and elemental analyses.

To determine the optimal reaction conditions for the efficient preparation of the target molecules, the reaction temperature was varied. The results indicated that room temperature should be employed during the addition of aroyl chloride to prevent the facile generation of anhydride. The target molecular structure depended on the molar ratio of compound 2 and aroyl chloride. When the molar ratio of compound 2 and aroyl chloride was less than 1:1.5, the product was primarily the mono-substitution product 4 . However, when the molar ratio was 1:2.2-1:2.5, both mono- and di-substituted products were generated. This finding is in good agreement with previous reports. According to the yields of 3 and 4, the substituents on the benzene ring exerted no significant effects. All of the 1,8-naphthalimide derivatives were confirmed via ${ }^{1} \mathrm{H}$ NMR, ${ }^{13} \mathrm{C}$ NMR, MS, and elemental analysis, and the data are reported in the experimental section.

To further confirm the structure of the synthesized compounds, a single-crystal structure of compound $\mathbf{3 f}$ was obtained by dissolving the crystal in ethanol followed by slow evaporation of the solvent at room temperature over approximately 3 days. The molecular structure of compound $\mathbf{3 f}$ is shown in Figure 1. A packing diagram is shown in Figure 2. The hydrogen bonds are shown as dashed lines. The core plane (naphthalimide ring) and the phenyl ring (C20, C21, C22, C23, C24, and C25) formed a dihedral angle of 37.8 ${ }^{\circ}$, and the other phenyl ring (C29, C30, C31, C32, C33, and C34) formed a dihedral angle of 46.1 ${ }^{\circ}$. The dihedral angle between the two phenyl rings $(\mathrm{C} 2, \mathrm{C} 3, \mathrm{C} 4, \mathrm{C} 5, \mathrm{C} 6$, and $\mathrm{C} 12$; $\mathrm{C} 6, \mathrm{C} 7, \mathrm{C} 8, \mathrm{C} 9, \mathrm{C} 10$, and C12) was 3.8 $\AA$, and the pyridine ring formed dihedral angles of $2.7 \AA$ and $4.2 \AA$ to the former two phenyl rings, respectively. These results showed that all of the atoms of the rings were almost in the same plane. The $3 \mathrm{D}$ net consists of moderate $\pi-\pi$ stacking interactions with a centroid-to-centroid distance of $3.6548 \AA$, and the shortest distance (X1A to C2, C3) between the core planes was $3.490 \AA$ (Figure 3).

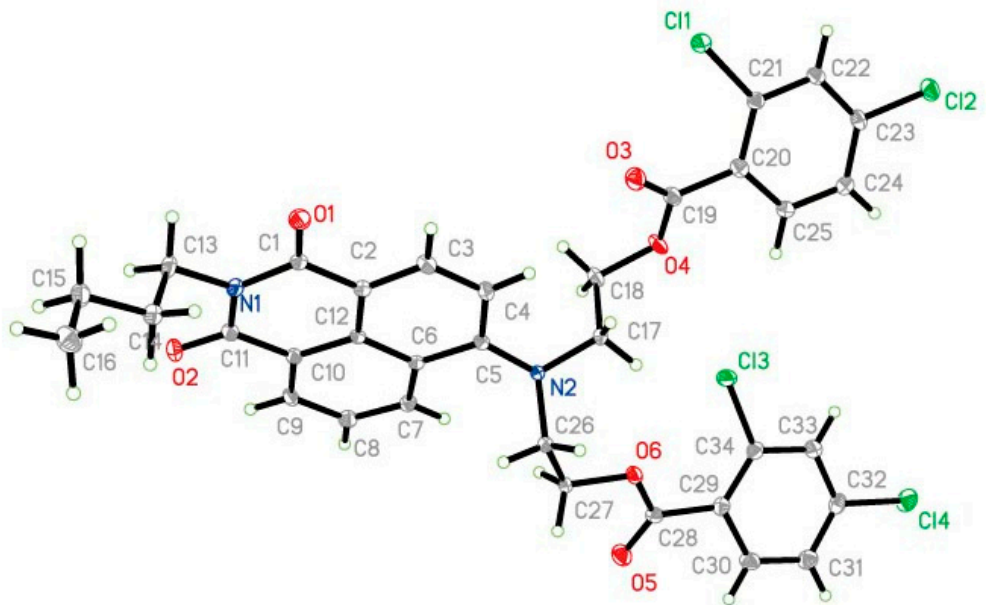

Figure 1. Molecular structure of $3 f$. 


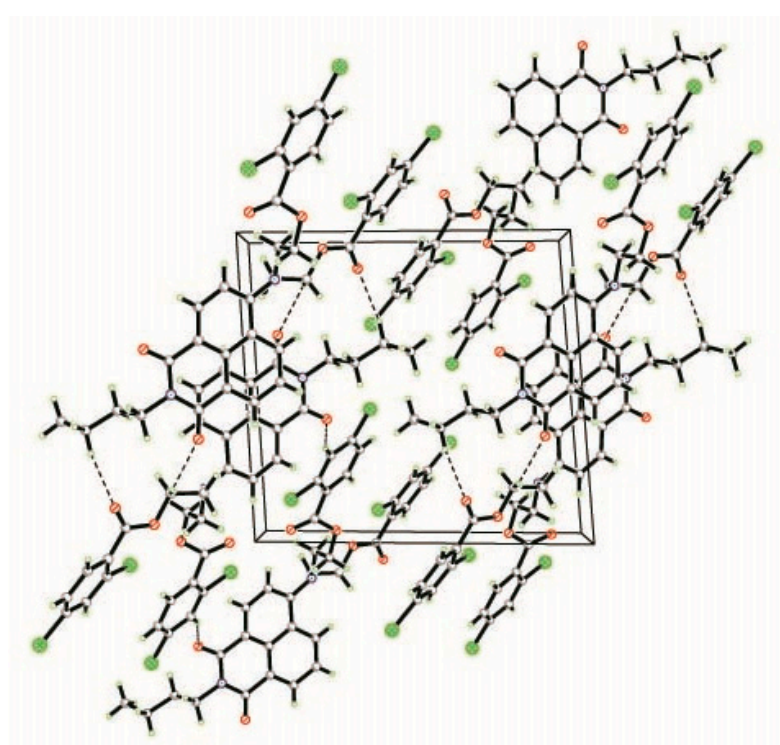

Figure 2. Packing view of $\mathbf{3 f}$.

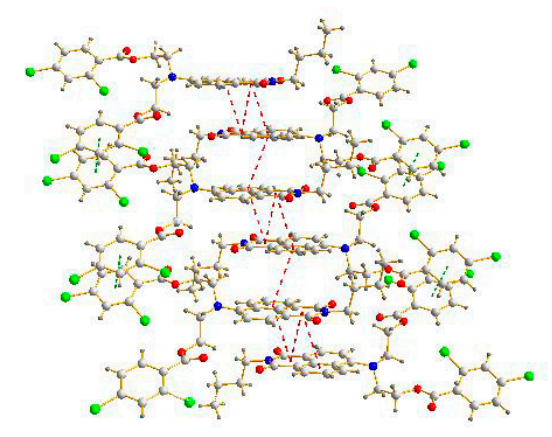

Figure 3. $\pi-\pi$ Stacking interactions between the core planes.

\subsection{Absorption spectra and fluorescence}

The photophysical properties of 1,8-naphthalimides mainly originate from the polarization of the naphthalimide moiety due to the electron donor-acceptor interaction occurring between the substituents at C-4 (electron donor) and the carbonyl groups from the imide structure (electron acceptor) of the molecule [31]. The normalized UV-vis spectra of compounds $3 \mathbf{a}-\mathbf{3 f}$ and $\mathbf{4 a}-\mathbf{4} \mathbf{f}$ in $\mathrm{EtOH} / \mathrm{H}_{2} \mathrm{O}(\mathrm{v}: \mathrm{v}=4: 1)$ with a concentration of $1 \times 10^{-5} \mathrm{M}$ are listed in Table 2. As shown in Table 2, the main absorption band of these dyes is centered between 414 and $440 \mathrm{~nm}$. No obvious difference is observed between mono- and di-substitution because the substituents at benzene minimally affected the parent scaffolds. No obvious effect was observed because benzoyl is too far from the conjugated system, as could be found in the single crystal structure. Although the molecular polarities of compounds $\mathbf{3}$ and $\mathbf{4}$ are different, both of the substitutions at C-4 are ethylamino, which result in similar electron donors to the parent scaffolds. 
Table 2. Absorption and fluorescence characteristics of compounds a-f in $\mathrm{EtOH} / \mathrm{H}_{2} \mathrm{O}$ (v:v=4:1) solution.

\begin{tabular}{|c|c|c|c|c|c|}
\hline Comp. & $\begin{array}{l}\log \varepsilon \\
\left(1 \mathrm{M}^{-1} \mathrm{~cm}^{-1}\right)^{\mathrm{a}}\end{array}$ & $\lambda \max ^{b}$ & A & $\lambda \mathrm{em}^{\mathrm{b}}$ & $\begin{array}{l}\text { Fluorescence } \\
\text { intensity }\end{array}$ \\
\hline $3 a$ & 4.286 & 421.5 & 0.193 & 521 & 177.99 \\
\hline $3 b$ & 3.748 & 419.5 & 0.056 & 529 & 66.17 \\
\hline $3 c$ & 4.100 & 420.0 & 0.126 & 524 & 125.93 \\
\hline $3 d$ & 3.699 & 414.0 & 0.05 & 522 & 68.21 \\
\hline $3 e$ & 4.336 & 417.0 & 0.217 & 527 & 202.28 \\
\hline $3 f$ & 4.017 & 421.5 & 0.104 & 528 & 134.29 \\
\hline $4 a$ & 4.241 & 439.5 & 0.175 & 526 & 389.54 \\
\hline $4 b$ & 4.255 & 439.5 & 0.180 & 524 & 398.44 \\
\hline $4 c$ & 4.233 & 440.5 & 0.171 & 523 & 369.22 \\
\hline $4 d$ & 4.140 & 440.0 & 0.138 & 523 & 329.4 \\
\hline $4 e$ & 4.201 & 440.0 & 0.159 & 522 & 361.9 \\
\hline $4 \mathrm{f}$ & 4.212 & 438.5 & 0.163 & 521 & 390.58 \\
\hline
\end{tabular}

a Extinction coefficient.

${ }^{\mathrm{b}}$ Maximum absorbance $\left(\lambda_{\mathrm{abs}}\right)$ and emission intensity $\left(\lambda_{\mathrm{em}}\right)$ wavelengths.

The emission was detected at 521-529 nm. Compared with that of compound $3 \mathbf{a}\left(\lambda_{\max }=521\right.$ $\mathrm{nm})$, the emission maxima of other bi-substituted compounds, namely $\mathbf{3 b} \mathbf{b}-\mathbf{3} \mathbf{f}$, were all slight red-shifted, which might the result of the electron-withdrawing and electron-donating capabilities of the substituents on the benzene ring. In addition, the emission maxima of mono-substituted compound were almost equal [32]. However, the mono-substitution exhibited better fluorescence intensity of approximately 329.4-398.44 nm, almost two-fold greater than that of the di-substitution, indicating that the mono-substituent exhibited a better fluorescence intensity than the di-substituent at the same concentration. This result indicated that the mono-substituted compounds might be the probe molecules due to their better fluorescence intensity and the large space between the hydroxyl and carbonyl.

\subsection{Solvent effect}


Compound 3f was selected for the fluorescence intensity experiment because its single crystal structure was determined by X-ray analysis, which was able to explain the ion-recognition performance based on the molecular configuration.

As shown in Figure 4, the fluorescence emission of compound $\mathbf{3 f}$ is more dependent on the solvent. As the solvent polarity increased, the emission wavelength $\lambda_{\mathrm{em}}$ was red-shifted, and the fluorescence intensity decreased. The fluorescence maximum shifts to longer wavelengths from nonpolar to polar solvents are indicative of the charge transfer nature of the emitting state. As shown in Figure4, the $\lambda_{\mathrm{em}}$ for $3 \mathrm{f}$ shifted from $495 \mathrm{~nm}$ to $530 \mathrm{~nm}$ as the solvent was changed from dichloromethane to dimethylformamide. Examination of the chemical structure of entitled dyes suggested that the occurrence of an intramolecular charge transfer (ICT) process might be responsible for their solvent sensitivity. Therefore, ethanol and water were selected as the solvent, thereby reducing the use of organic solvents and allowing the dissolution of metal ions, which are poorly soluble in organic solvents.

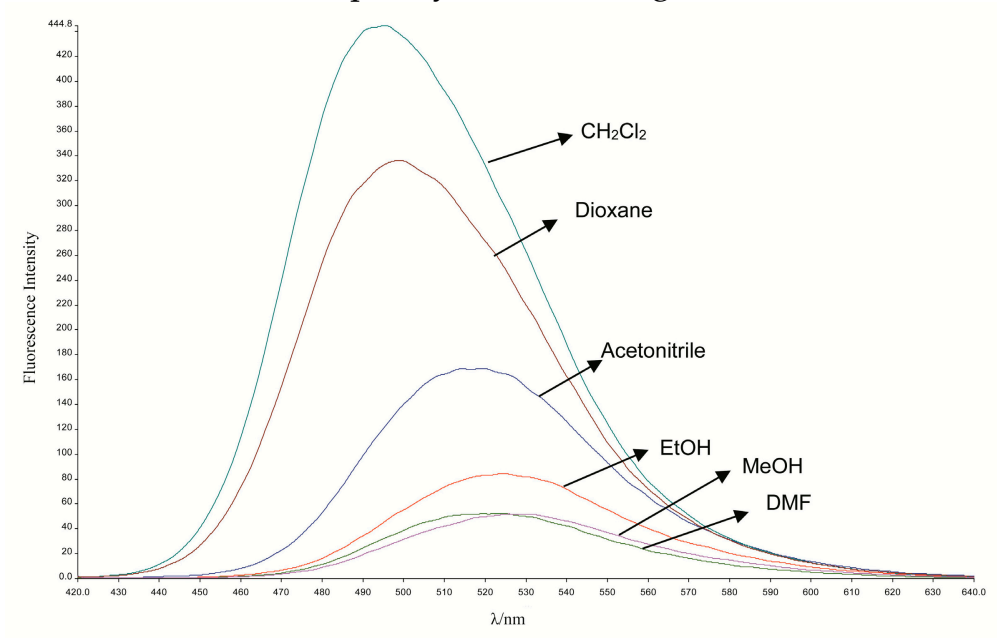

Figure 4. Various solvents' influences on the fluorescence intensity.

\subsection{Selectivity of probe $3 f$}

Figure 5 shows the normalized emission spectra of compound $3 f$ in $\mathrm{EtOH} / \mathrm{H}_{2} \mathrm{O}$ (v:v=4:1) solution $\left(1 \times 10^{-5} \mathrm{M}\right)$ that was excited at its absorption maximum. As shown in Figure 5 , the fluorescence intensity was lower than that of other ions when $\mathrm{Pb}^{2+}$ or $\mathrm{Hg}^{2+}$ was added, indicating that compound $3 \mathrm{f}$ might be a probe molecule for $\mathrm{Pb}^{2+}$. The fluorescence intensity difference may be attributable to the formation of a compound $3 \mathrm{f}-\mathrm{Pb}^{2+}$ complex; the possible structure of this complex is proposed in Figure 6.

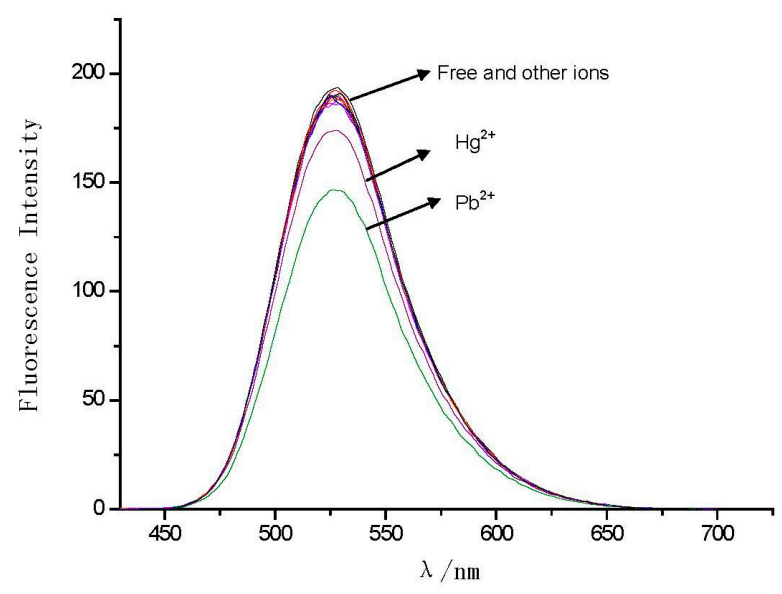


Figure 5. Fluorescence intensity response of probe $3 \mathrm{f}$ to different metal cations.

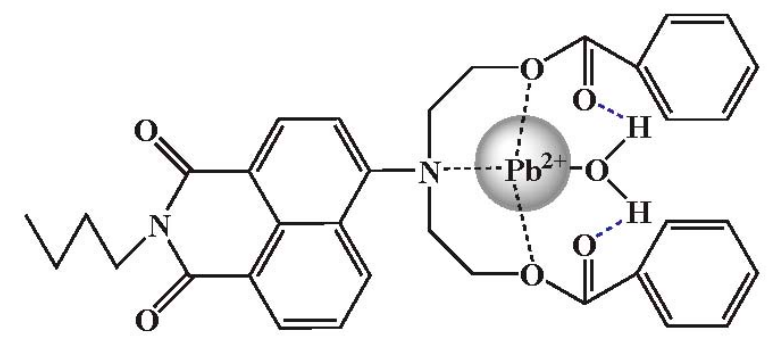

Figure 6. Proposed structure of the $3 \mathrm{f}-\mathrm{Pb}^{2+}$ complex.

As shown in Figure 7, as the $\mathrm{Pb}^{2+}$ concentration increased, the absorption maximum shifted to shorter wavelengths, and the emission intensity decreased significantly with an increase in the $\mathrm{Pb}^{2+}$ concentration. These results can contribute to the design of new fluorescence materials based on 1,8-naphthalimide derivatives.

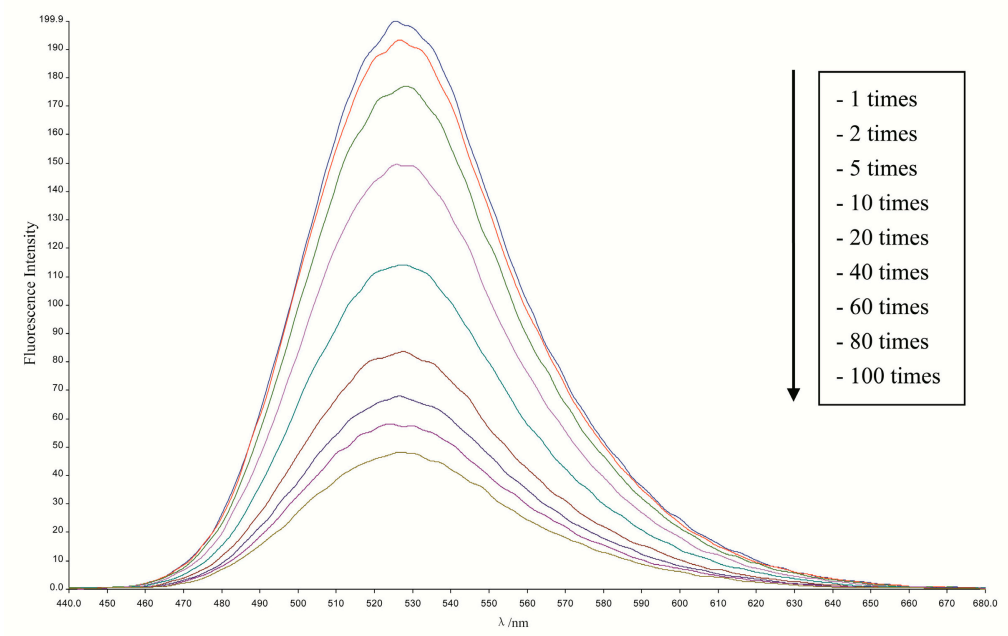

Figure 7. Fluorescence spectra of the $3 \mathrm{f}$ probe under the same conditions except for the addition of

different amounts of $\mathrm{Pb}^{2+}$.

\section{Experimental}

\subsection{Chemicals and instruments}

All of the solvents and reactants are commercially available and were used without purification. The melting points were determined using a Beijing Taike melting point apparatus $(\mathrm{X}-4)$ and were uncorrected. The ${ }^{1} \mathrm{H}$ NMR and ${ }^{13} \mathrm{C}$ NMR spectra were recorded on a Bruker AVANCE $300 \mathrm{MHz}$ or $400 \mathrm{MHz}$ nuclear magnetic resonance spectrometer using $\mathrm{CDCl}_{3}$ or DMSO- $d_{6}$ as the solvent and TMS as the internal standard. The mass spectra were recorded on a Waters XevoTQ mass spectrometer. The elemental analysis was performed on FLASH EA1112 elemental analyzer. Absorption spectra were collected using a PERSEE TU-1900 ultraviolet spectrophotometer. Fluorescence emission spectra were obtained on a Perkin Elmer LS55 fluorospectrophotometer. The X-ray data were collected on a Bruker AXSII CCD 
area-detector diffractometer using graphite monochromated Mo Ka radiation $(\lambda=0.71073 \AA)$ at 293(2) K. All of the calculations were performed with the SHELX-97 program package $[24,25]$.

\subsection{Synthesis of N-n-butyl-4-bromo-1,8-naphthalimide (1)}

$\mathrm{N}$-n-Butyl-4-bromo-1,8-naphthalimide was prepared by modifying a previously reported procedure [26] to obtain an improved yield of $84 \%$. 4-Bromo-1,8-naphthalic anhydride (58 mmol, $16.1 \mathrm{~g})$ and n-butylamine $(60 \mathrm{mmol}, 4.4 \mathrm{~g})$ were heated under reflux in ethanol $(250 \mathrm{~mL})$ with vigorous stirring for $12 \mathrm{~h}$ under $\mathrm{N}_{2}$. Then, the mixture was cooled, and the precipitated solids were filtered and recrystallized from ethanol to yield $13.5 \mathrm{~g}(70 \%)$ of a light-yellow product. mp 105-106 ${ }^{\circ} \mathrm{C}$; ${ }^{1} \mathrm{H}$ NMR (DMSO- $d_{6}, 300 \mathrm{MHz}$ ), $\delta$ (ppm): 7.92-8.52 (m, 5H), 3.98-4.03 (t, $J=7.3 \mathrm{~Hz}, 2 \mathrm{H}), 1.56-1.66(\mathrm{~m}, 2 \mathrm{H}), 1.31-1.39(\mathrm{~m}, 2 \mathrm{H}), 0.90-0.95(\mathrm{t}, J=7.3 \mathrm{~Hz}, 3 \mathrm{H})$. Anal. Calcd. For $\mathrm{C}_{16} \mathrm{H}_{14} \mathrm{BrNO}_{2}(\%): \mathrm{C}, 57.85 ; \mathrm{H}, 4.25 ; \mathrm{N}, 4.22$. Found: C, 57.80; H, 4.18; N, 4.28.

\subsection{Synthesis of $N$-n-butyl-4- $N$ ', $N$-dihydroxyethyl-1,8-naphthalimide (2)}

N-n-Butyl-4-(N',N'-dihydroxyethyl)amino-1,8-naphthalimide was obtained using a procedure similar to that reported by Guo et al. [27]. N-n-Butyl-4-bromine-1,8-naphthalimide $(45.2 \mathrm{mmol}, 15 \mathrm{~g})$ and diethanolamine $(75 \mathrm{~mL} \mathrm{mmol})$ were mixed in ethylene glycol monomethyl ether $(100 \mathrm{~mL})$. The mixture was refluxed for $6 \mathrm{~h}$. The crude product was purified by column chromatography using silica gel, with an EtOAc and light petroleum (v:v=1:4) solution as the eluent. $N$-n-Butyl-4-( $N^{`}, N^{\prime}$-dihydroxyethyl)amino-1,8-naphthalene imide (BNI) was obtained as a yellow solid at a yield of $20.4 \%$. mp $129-1300^{\circ} \mathrm{C}$; ${ }^{1} \mathrm{H}$ NMR $(400 \mathrm{MHz}$, $\left.\mathrm{CDCl}_{3}\right) \delta(\mathrm{ppm})$ 8.88-7.28 (m, 5H), 4.16-4.12 (t, J=7.2 Hz, 2H), 3.85-3.88 (t, J=5.2 Hz, 4H), 3.61-3.64 (t, J=5.2 Hz, $4 \mathrm{H}), 2.81(\mathrm{~s}, 2 \mathrm{H}), 1.68-1.69(\mathrm{~m}, 2 \mathrm{H}), 1.43-1.45(\mathrm{~m}, 2 \mathrm{H}), 0.96-0.99(\mathrm{t}, J=7.2 \mathrm{~Hz}$, 3H). ${ }^{13} \mathrm{C}$ NMR $\left(100 \mathrm{MHz}, \mathrm{CDCl}_{3}\right) \delta$ (ppm) 164.34, 163.91, 154.28, 131.18, 131.22, 130.85, 130.14, 127.24, 125.65, 122.92, 117.18, 117.05, 59.73, 59.73, 55.33, 55.33, 40.10, 30.20, 20.38, 13.85. Anal. Calcd. for $\mathrm{C}_{20} \mathrm{H}_{24} \mathrm{~N}_{2} \mathrm{O} 4$ (\%): C 67.40; H 6.79; N 7.86. Found: C 67.28; H 6.86; N 7.95.

\subsection{General procedure for the synthesis of $N$-n-butyl-4-[N`,N`-dihydroxyethyl]-1,8-naphthalene imide}

A mixture of BNI $(1.0 \mathrm{~g}, 2.81 \mathrm{mmol})$ and TEA $(0.63 \mathrm{~g}, 6.25 \mathrm{mmol})$ solution in dichloromethane $(40 \mathrm{~mL})$ was stirred, and substituted benzoyl chloride $(6.25 \mathrm{mmol})$ was then slowly added over $30 \mathrm{~min}$ and allowed to react for $8 \mathrm{~h}$ at $25^{\circ} \mathrm{C}$. The product was neutralized by saturated $\mathrm{Na}_{2} \mathrm{CO}_{3}(\mathrm{aq})$ and purified by column chromatography on a silica gel column using cyclohexane-EtOAc (v:v=1:3) as the eluent.

\subsection{1 [(2-Butyl-1,3-dioxo-2,3-dihydro-1H-benzo[de]isoquinolin-6-yl)imino]diethane-2,1-diyl} dibenzoate (3a)

Yield 46.0\%, yellow solid, mp 148-150 ${ }^{\circ}$; IR $\left(\mathrm{KBr}\right.$, wit $\left./ \mathrm{cm}^{-1}\right)$ 3059-2897 (C-H), $1719(\mathrm{C}=\mathrm{O})$. ${ }^{1} \mathrm{H}$ NMR $\left(400 \mathrm{MHz}, \mathrm{CDCl}_{3}\right) \delta(\mathrm{ppm})$ 8.53-7.28 (m, 15H), 4.54-4.51 (t, J=5.2 Hz, 4H), 4.17-4.13 (t, $J=7.2 \mathrm{~Hz}, 2 \mathrm{H}), 3.91-3.88(\mathrm{t}, J=5.2 \mathrm{~Hz}, 4 \mathrm{H}), 1.75-1.67(\mathrm{~m}, 2 \mathrm{H}), 1.50-1.41(\mathrm{~m}, 2 \mathrm{H}), 1.01-0.97(\mathrm{t}, J=7.8$ $\mathrm{Hz}, 3 \mathrm{H}) .{ }^{13} \mathrm{C}$ NMR $\left(100 \mathrm{MHz}, \mathrm{CDCl}_{3}\right) \delta(\mathrm{ppm}) 166.15,166.15,164.28,163.64,153.96,153.96$, $133.13,133.13,131.74,131.19,130.26,129.93,129.51,129.42,128.27,128.27,128.27,128.27$, $127.61,127.61,125.92,125.92,123.92,123.20,118.85,117.69,62.06,62.06,52.76,52.76,40.03$, 30.24, 20.39, 13.88. MS (ESI) $m / z$ : $565(\mathrm{M}+\mathrm{H})^{+}$. Anal. Calcd. for $\mathrm{C}_{34} \mathrm{H}_{32} \mathrm{~N}_{2} \mathrm{O}_{6}(\%)$ : C 72.32; $\mathrm{H} 5.71 ; \mathrm{N}$ 4.96. Found: C 72.44; H 5.68; N 4.82.

3.4.2 [(2-Butyl-1,3-dioxo-2,3-dihydro-1H-benzo[de]isoquinolin-6-yl)imino]diethane-2,1-diyl bis(2-methylbenzoate) $(3 b)$ 
Yield 48.2\%, yellow solid, mp 94-95 ${ }^{\circ}$; IR (KBr, $\left.\widetilde{v} / \mathrm{cm}^{-1}\right)$ 2959-2871 (C-H), $1717(\mathrm{C}=\mathrm{O}) .{ }^{1} \mathrm{H}$ NMR $\left(300 \mathrm{MHz}, \mathrm{CDCl}_{3}\right) \delta(\mathrm{ppm})$ 8.54 7.08 (m, 13H) 4.51-4.47 (t, J=5.4 Hz, 4H), 4.17-4.12 (t, $J=7.5 \mathrm{~Hz}, 2 \mathrm{H}), 3.96-3.87(\mathrm{t}, J=5.4 \mathrm{~Hz}, 4 \mathrm{H}), 2.46(\mathrm{~s}, 6 \mathrm{H}), 1.74-1.66(\mathrm{~m}, 2 \mathrm{H}), 1.49-1.42(\mathrm{~m}, 2 \mathrm{H})$, 1.02-0.97 (t, 3H). ${ }^{13} \mathrm{C}$ NMR $\left(75 \mathrm{MHz}, \mathrm{CDCl}_{3}\right) \delta(\mathrm{ppm})$ 166.96, 166.96, 164.38, 163.72, 153.96, $140.52,140.52,132.28,132.28,131.76,131.76,131.25,130.47,130.47,130.31,129.99,128.68$, $128.68,127.55,125.92,125.92,125.59,125.59,123.27,118.70,117.67,61.68,61.68,52.83,52.83$, 40.12, 30.30, 21.75, 21.75, 20.46, 13.93. Anal. Calcd. for $\mathrm{C}_{36} \mathrm{H}_{36} \mathrm{~N}_{2} \mathrm{O}_{6}$ (\%): C 72.95; H 6.12; N 4.73. Found: C 72.98; H 6.21; N 4.78 .

3.4.3 [(2-Butyl-1,3-dioxo-2,3-dihydro-1H-benzo[de]isoquinolin-6-yl)imino]diethane-2,1-diyl bis(4-methylbenzoate) (3c)

Yield 30.4\%, yellow solid, mp 104-105 ${ }^{\circ} \mathrm{C}$; IR (KBr, $\left.\widetilde{v} / \mathrm{cm}^{-1}\right)$ 2957-2927 (C-H), 1707 (C=O). ${ }^{1} \mathrm{H}$ NMR $\left(400 \mathrm{MHz}, \mathrm{CDCl}_{3}\right) \delta(\mathrm{ppm})$ 8.56-7.13 (m, 13H), 4.53-4.51 (t, J=5.6 Hz, 2H), 4.20-4.17 (t, $J=7.6 \mathrm{~Hz}, 2 \mathrm{H}), 3.91-3.88(\mathrm{t}, J=5.2 \mathrm{~Hz}, 2 \mathrm{H}), 3.57-3.56(\mathrm{~m}, 1 \mathrm{H}), 3.31-3.30(\mathrm{~m}, 1 \mathrm{H}), 2.45-2.38(\mathrm{~m}, 8 \mathrm{H})$, 1.76-1.75 (m, 1H), 1.74-1.39 (m, 7H). $\left.{ }^{13} \mathrm{C} \mathrm{NMR} \mathrm{(100} \mathrm{MHz,} \mathrm{CDCl}_{3}\right) \delta(\mathrm{ppm}) 171.88,171.88,166.32$, $164.41,163.76,154.15,154.15,144.49,143.91,139.17,134.15,134.15,130.24,130.24,130.24$, 130.24, 129.51, 129.51, 129.19, 129.19, 129.01, 129.01, 126.76, 126.76, 126.41, 118.84, 61.94, 61.94, 52.79, 52.79, 40.09, 30.33, 21.76, 21.76, 20.42, 13.89. MS (ESI) m/z: $593(\mathrm{M}+\mathrm{H})^{+}$. Anal. Calcd. for $\mathrm{C}_{36} \mathrm{H}_{36} \mathrm{~N}_{2} \mathrm{O}_{6}(\%): \mathrm{C} 72.95 ; \mathrm{H}$ 6.12; N 4.73. Found: C 72.79; H 6.25; N 4.82.

3.4.4 [(2-Butyl-1,3-dioxo-2,3-dihydro-1H-benzo[de]isoquinolin-6-yl)imino]diethane-2,1-diyl bis(4-chlorobenzoate) (3d)

Yield 33.7\%, yellow solid, $\mathrm{mp} 88-90^{\circ} \mathrm{C}$; IR (KBr, $\left.\widetilde{v} / \mathrm{cm}^{-1}\right)$ 2957-2871 (C-H), 1718, 1690, 1664 $(\mathrm{C}=\mathrm{O}) .{ }^{1} \mathrm{H}$ NMR $\left(400 \mathrm{MHz}, \mathrm{CDCl}_{3}\right) \delta(\mathrm{ppm})$ 8.56-7.28 (m, $\left.13 \mathrm{H}\right) 4.52-4.49(\mathrm{t}, J=5.4 \mathrm{~Hz}, 4 \mathrm{H})$, 4.19-4.11 (m, 2H), 3.88-3.84 (t, J=5.4 Hz, 4H), 1.74-1.68 (m, 2H), 1.51-1.41 (m, 2H), 1.01-0.96 (t, $J=7.2 \mathrm{~Hz}, 3 \mathrm{H}) .{ }^{13} \mathrm{C}$ NMR $\left(100 \mathrm{MHz}, \mathrm{CDCl}_{3}\right) \delta(\mathrm{ppm}) 165.35,164.25,163.62,153.73,139.80,139.80$, $131.72,131.35,130.82,130.82,130.82,130.82,130.09,129.97,128.71,128.71,128.71,128.71$, 127.93, 127.93, 127.70, 126.09, 126.09, 123.34, 118.90, 117.99, 62.25, 62.25, 52.82, 52.82, 40.21, 30.33, 20.46, 13.91. MS (ESI) $m / z: 633(\mathrm{M}+\mathrm{H})^{+}$. Anal. Calcd. for $\mathrm{C}_{34} \mathrm{H}_{30} \mathrm{Cl}_{2} \mathrm{~N}_{2} \mathrm{O}_{6}(\%)$ : C 64.46; $\mathrm{H}$ 4.77; N 4.42. Found: C 64.55; H 4.71; N 4.38.

3.4.5 [(2-Butyl-1,3-dioxo-2,3-dihydro-1H-benzo[de]isoquinolin-6-yl)imino]diethane-2,1-diyl bis[4-(trifluoromethyl)benzoate] (3e)

Yield 20.8\%, yellow solid, mp 100-101 ${ }^{\circ} \mathrm{C}$; IR (KBr, $\left.\widetilde{v} / \mathrm{cm}^{-1}\right)$ 2960-2926 (C-H), $1731(\mathrm{C}=\mathrm{O})$. ${ }^{1} \mathrm{H}$ NMR $\left(400 \mathrm{MHz}, \mathrm{CDCl}_{3}\right) \delta(\mathrm{ppm})$ 8.58-8.46 (m, 2H), 7.91-7.89 (t, J=4.0 Hz, 3H), 7.62-7.48 (m, $5 \mathrm{H}), 4.58-4.55(\mathrm{t}, J=5.6 \mathrm{~Hz}, 3 \mathrm{H}), 4.18-4.15(\mathrm{t}, J=7.6 \mathrm{~Hz}, 2 \mathrm{H}), 3.92-3.89(\mathrm{t}, J=5.2 \mathrm{~Hz}, 3 \mathrm{H}), 1.49-1.28$ $(\mathrm{m}, 4 \mathrm{H}), 1.01-0.98(\mathrm{~m}, 8 \mathrm{H}) .{ }^{13} \mathrm{C}$ NMR $\left(100 \mathrm{MHz}, \mathrm{CDCl}_{3}\right) \delta(\mathrm{ppm}) 164.97,164.97,164.13,163.54$, $163.54,153.51,153.51,134.89,134.57,132.70,132.70,131.65,131.65,131.37,129.82,129.82$, $129.82,129.82,127.79,126.14,126.14,125.38,125.38,125.34,125.34,123.39,119.01,118.20,62.54$, 62.54, 52.82, 52.82, 40.11, 30.21, 20.39, 13.80. MS (ESI) $m / z$ : $701(\mathrm{M}+\mathrm{H})^{+}$. Anal. Calcd. for $\mathrm{C}_{36} \mathrm{H}_{30} \mathrm{~F}_{6} \mathrm{~N}_{2} \mathrm{O}_{6}$ (\%): C 61.71; H 4.32; N 4.00. Found: C 61.65; H 4.44; N 4.08.

3.4.6 [(2-Butyl-1,3-dioxo-2,3-dihydro-1H-benzo[de]isoquinolin-6-yl)imino]diethane-2,1-diyl bis(2,4-dichlorobenzoate) (3f)

Yield, 65.0\%, yellow solid, $\mathrm{mp}$ 87-88 ${ }^{\circ} \mathrm{C}$; IR (KBr, $\left.\widetilde{v} / \mathrm{cm}^{-1}\right)$ : 2961-2866 (C-H), 1731, 1710, $1652(\mathrm{C}=\mathrm{O}) .{ }^{1} \mathrm{H}$ NMR $\left(300 \mathrm{MHz}, \mathrm{CDCl}_{3}\right) \delta(\mathrm{ppm}) 8.58-7.15(\mathrm{~m}, 11 \mathrm{H}) 4.54-4.5(\mathrm{t}, J=5.4 \mathrm{~Hz}, 4 \mathrm{H})$, $4.20-4.15(\mathrm{t}, J=7.5 \mathrm{~Hz}, 2 \mathrm{H}), 3.91-3.87(\mathrm{t}, J=5.4 \mathrm{~Hz}, 4 \mathrm{H}), 1.77-1.67(\mathrm{~m}, 2 \mathrm{H}), 1.50-1.42(\mathrm{~m}, 2 \mathrm{H})$, 1.02-0.97 (m, J=7.2 Hz, 3H). ${ }^{13} \mathrm{C}$ NMR $\left(75 \mathrm{MHz}, \mathrm{CDCl}_{3}\right) \delta(\mathrm{ppm}) 164.28,164.28,163.61,163.61$, $153.58,138.80,135.05,135.05,132.44,132.44,132.44,131.67,131.39,131.15,131.15,131.15$, $130.12,129.95,12.95,127.58,127.40,126.92,126.15,123.34,118.82,117.95,62.57,62.57,52.62$, 
52.62, 40.19, 30.33, 20.45, 13.91. MS (ESI) $m / z$ : $703(\mathrm{M}+\mathrm{H})^{+}$. Anal. Calcd. for $\mathrm{C}_{34} \mathrm{H}_{28} \mathrm{Cl}_{4} \mathrm{~N}_{2} \mathrm{O}_{6}(\%)$ : C 58.14; H 4.02; N 3.99. Found: C 58.26; H 3.95; N 3.89.

\subsection{7}

2-[(2-butyl-1,3-dioxo-2,3-dihydro-1H-benzo[de]isoquinolin-6-yl)-(2-hydroxy-ethyl)-amino]-et hyl ester (4a)

Yield 61.4\%, yellow solid, mp 158-159 ${ }^{\circ}$; IR $\left(\mathrm{KBr}, \widetilde{v} / \mathrm{cm}^{-1}\right) 3396(\mathrm{O}-\mathrm{H}), 2965-2820(\mathrm{C}-\mathrm{H})$, $1720(\mathrm{C}=\mathrm{O}) .{ }^{1} \mathrm{H}$ NMR $\left(400 \mathrm{MHz}, \mathrm{CDCl}_{3}\right) \delta(\mathrm{ppm}) 8.55-7.45(\mathrm{~m}, 10 \mathrm{H}), 6.71(\mathrm{~d}, J=8.4 \mathrm{~Hz}, 1 \mathrm{H}), 6.01$ $(\mathrm{s}, 1 \mathrm{H}), 4.78(\mathrm{t}, J=10.4 \mathrm{~Hz}, 2 \mathrm{H}), 4.14(\mathrm{t}, J=11.2 \mathrm{~Hz}, 4 \mathrm{H}), 3.78(\mathrm{t}, J=10.4 \mathrm{~Hz}, 2 \mathrm{H}), 2.03(\mathrm{~s}, 2 \mathrm{H}), 1.69(\mathrm{t}$, $J=15.2 \mathrm{~Hz}, 2 \mathrm{H}), 1.24(\mathrm{~s}, 2 \mathrm{H}), 0.95(\mathrm{t}, J=14.8 \mathrm{~Hz}, 3 \mathrm{H}) .{ }^{13} \mathrm{C} \mathrm{NMR}\left(100 \mathrm{MHz}, \mathrm{CDCl}_{3}\right) \delta(\mathrm{ppm}) 167.83$, $164.64,164.13,164.13,149.04,149.04,134.24,133.62,131.14,131.14,129.81,129.35,128.58$, $128.58,126.07,125.03,123.19,120.41,111.06,63.17,60.40,60.16,43.98,40.02,30.33,20.45,13.89$. MS (ESI) $m / z$ : $461(\mathrm{M}+\mathrm{H})^{+}$. Anal. Calcd. for $\mathrm{C}_{27} \mathrm{H}_{28} \mathrm{~N}_{2} \mathrm{O}_{5}$ (\%): $\mathrm{C}$ 70.42; $\mathrm{H}$ 6.13; N 6.08. Found: $\mathrm{C}$ 70.34; H 6.25; N 6.03 .

\section{4 .8}

2-[(2-Butyl-1,3-dioxo-2,3-dihydro-1H-benzo[de]isoquinolin-6-yl)(2-hydroxyethyl)amino]ethyl -2-methylbenzoate (4b)

Yield 57.6\%, yellow solid, mp 179-180 ${ }^{\circ}$; IR $\left(\mathrm{KBr}, \widetilde{v} / \mathrm{cm}^{-1}\right) 3367(\mathrm{OH}), 2957-2927(\mathrm{C}-\mathrm{H})$, $1717(\mathrm{C}=\mathrm{O}) .{ }^{1} \mathrm{H}$ NMR $\left(400 \mathrm{MHz}, \mathrm{CDCl}_{3}\right) \delta(\mathrm{ppm})$ 8.60-7.37 (m, 9H), 6.76-6.74 (d, J=8.0 Hz, $\left.1 \mathrm{H}\right)$, $5.31(\mathrm{~s}, 1 \mathrm{H}), 4.80(\mathrm{t}, 2 \mathrm{H}), 4.18-4.16(\mathrm{t}, J=6.8 \mathrm{~Hz}, 4 \mathrm{H}), 3.81(\mathrm{t}, 2 \mathrm{H}), 2.42(\mathrm{~d}, J=8.4 \mathrm{~Hz}, 3 \mathrm{H}), 1.73-1.71$ $(\mathrm{d}, J=6.8 \mathrm{~Hz}, 2 \mathrm{H}), 1.47-1.45(\mathrm{~m}, 2 \mathrm{H}), 1.27(\mathrm{t}, 3 \mathrm{H}), 0.98(\mathrm{t}, 3 \mathrm{H}) .{ }^{13} \mathrm{C} \mathrm{NMR}\left(100 \mathrm{MHz}, \mathrm{CDCl}_{3}\right) \delta$ (ppm) 171.66, 168.02, 164.68, 164.17, 149.13, 138.44, 138.29, 134.48, 131.16, 131.16, 130.69, 128.37, 126.95, 126.17, 125.00, 123.16, 120.42, 110.98, 104.11, 63.12, 43.96, 43.96, 40.04, 30.33, 29.72, 21.27, 20.45, 13.90. MS (ESI) $m / z$ : $475(\mathrm{M}+\mathrm{H})^{+}$. Anal. Calcd. for $\mathrm{C}_{28} \mathrm{H}_{30} \mathrm{~N}_{2} \mathrm{O}_{5}$ (\%): C 70.87; H 6.37; N 5.90. Found: C 70.75; H 6.44; N 5.92.

\subsection{9}

2-[(2-Butyl-1,3-dioxo-2,3-dihydro-1H-benzo[de]isoquinolin-6-yl)(2-hydroxyethyl)amino]ethyl -4-methylbenzoate (4c)

Yield 61.2\%, yellow solid, mp 168-169 ${ }^{\circ} \mathrm{C}$; IR $\left(\mathrm{KBr}, \widetilde{v} / \mathrm{cm}^{-1}\right) 3362(\mathrm{O}-\mathrm{H}), 2957-2825(\mathrm{C}-\mathrm{H})$, $1704(\mathrm{C}=\mathrm{O}) .{ }^{1} \mathrm{H}$ NMR $\left(400 \mathrm{MHz}, \mathrm{CDCl}_{3}\right) \delta(\mathrm{ppm}) 8.59-7.25(\mathrm{~m}, 9 \mathrm{H}), 4.81-4.78(\mathrm{t}, J=5.2 \mathrm{~Hz}, 2 \mathrm{H})$, $4.20-4.13(\mathrm{t}, J=7.6 \mathrm{~Hz}, 3 \mathrm{H}), 3.81-3.79(\mathrm{t}, J=5.2 \mathrm{~Hz}, 2 \mathrm{H}), 2.45-2.43(\mathrm{~d}, J=8.8 \mathrm{~Hz}, 3 \mathrm{H}), 2.07(\mathrm{~s}, 1 \mathrm{H})$, 1.75-1.74 (m, 2H), $1.45\left(\mathrm{~m}, 2 \mathrm{H}, \mathrm{CH}_{2}\right), 0.95(\mathrm{t}, J=14.8 \mathrm{~Hz}, 3 \mathrm{H}) .{ }^{13} \mathrm{C} \mathrm{NMR}\left(100 \mathrm{MHz}, \mathrm{CDCl}_{3}\right) \delta$ (ppm) 171.28, 167.94, 164.67, 164.17, 149.13, 144.49, 134.30, 131.14, 131.14, 130.23, 130.23, 129.85, 129.29, 126.15, 125.00, 123.16, 120.41, 110.96, 104.06, 63.01, 60.42, 60.42, 44.03, 40.03, 30.37, 21.72, 20.46, 13.90. MS (ESI) $m / z: 475(\mathrm{M}+\mathrm{H})^{+}$. Anal. Calcd. for $\mathrm{C}_{28} \mathrm{H}_{30} \mathrm{~N}_{2} \mathrm{O}_{5}$ (\%): C 70.87; H 6.37; N 5.90. Found: C 70.72; H 6.48; N 5.95 .

3.4.10

2-[(2-Butyl-1,3-dioxo-2,3-dihydro-1H-benzo[de]isoquinolin-6-yl)(2-hydroxyethyl)amino]ethyl -4-chlorobenzoate (4d)

Yield 60.5\%, yellow solid, mp 167-168 ${ }^{\circ} \mathrm{C}$; IR $\left(\mathrm{KBr}, \widetilde{v} / \mathrm{cm}^{-1}\right) 3361(\mathrm{O}-\mathrm{H}), 2958-2927(\mathrm{C}-\mathrm{H})$, $1720(\mathrm{C}=\mathrm{O}) .{ }^{1} \mathrm{H}$ NMR $\left(400 \mathrm{MHz}, \mathrm{CDCl}_{3}\right) \delta(\mathrm{ppm})$ 8.53-7.41 (m, 9H), 6.71-6.69 (d, J=8.4 Hz, 1H), $5.98(\mathrm{~s}, 1 \mathrm{H}), 4.77-4.75(\mathrm{t}, J=4.8 \mathrm{~Hz}, 2 \mathrm{H}), 4.14-4.11(\mathrm{t}, J=8.4 \mathrm{~Hz}, 4 \mathrm{H}), 3.80-3.77(\mathrm{t}, J=5.2 \mathrm{~Hz}, 2 \mathrm{H}), 2.03$ $(\mathrm{s}, 1 \mathrm{H}), 1.70(\mathrm{~m}, 2 \mathrm{H}), 1.42(\mathrm{~m}, 2 \mathrm{H}), 0.95-0.91(\mathrm{t}, J=6.4 \mathrm{~Hz}, 3 \mathrm{H}) .{ }^{13} \mathrm{C} \mathrm{NMR}\left(100 \mathrm{MHz}, \mathrm{CDCl}_{3}\right) \delta$ (ppm) 166.84, 164.59, 164.08, 164.08, 149.00, 140.13, 134.18, 131.14, 131.14, 129.68, 128.92, 128.92, 127.78, 126.05, 125.01, 123.17, 120.40, 111.07, 104.08, 63.39, 60.41, 43.80, 40.02, 30.33, 20.45, 14.22, 13.89. MS (ESI) $m / z$ : $495(\mathrm{M}+\mathrm{H})^{+}$. Anal. Calcd. for $\mathrm{C}_{27} \mathrm{H}_{27} \mathrm{~N}_{2} \mathrm{O}_{5}$ (\%): C 65.52; H 5.50; N 5.66. Found: C 65.59; H 5.42; N 5.72. 


\subsubsection{1}

2-[(2-Butyl-1,3-dioxo-2,3-dihydro-1H-benzo[de]isoquinolin-6-yl)(2-hydroxyethyl)amino]ethyl -4-(trifluoromethyl)benzoate (4e)

Yield 50.4\%, yellow solid, mp 161-162 ${ }^{\circ}$; IR $\left(\mathrm{KBr}, \widetilde{v} / \mathrm{cm}^{-1}\right) 3389(\mathrm{O}-\mathrm{H}), 2886-2775(\mathrm{C}-\mathrm{H})$, $1715(\mathrm{C}=\mathrm{O}) .{ }^{1} \mathrm{H}$ NMR $\left(400 \mathrm{MHz}, \mathrm{CDCl}_{3}\right) \delta(\mathrm{ppm})$ 8.56-7.62 (m, 9H), 6.75-6.73 (d, J=4.8 Hz, $\left.1 \mathrm{H}\right)$, 5.96-5.95 (m, 1H), 4.82-4.80 (t, J=4.8 Hz, 2H), 4.16-4.12 (t, J=7.2 Hz, 4H), 3.84-3.81 (t, J=5.2 Hz, $2 \mathrm{H}), 2.03(\mathrm{~s}, 1 \mathrm{H}), 1.71-1.67(\mathrm{~m}, 2 \mathrm{H}), 1.45-1.39(\mathrm{~m}, 2 \mathrm{H}), 1.26(\mathrm{~s}, 1 \mathrm{H}), 0.97-0.93(\mathrm{t}, J=7.2 \mathrm{~Hz}, 3 \mathrm{H}) .{ }^{13} \mathrm{C}$ NMR (100 MHz, $\left.\mathrm{CDCl}_{3}\right) \delta$ (ppm) 166.47, 164.58, 164.09, 164.09, 148.98, 134.17, 133.70, 133.14, 131.22, 131.12, 131.12, 129.69, 129.40, 126.82, 126.13, 125.00, 123.16, 120.47, 111.14, 104.20, 63.48, 63.48, 63.48, 43.39, 40.01, 30.34, 20.45, 13.90. MS (ESI) $m / z$ : $529(\mathrm{M}+\mathrm{H})^{+}$. Anal. Calcd. for $\mathrm{C}_{28} \mathrm{H}_{27} \mathrm{~F}_{3} \mathrm{~N}_{2} \mathrm{O}_{5}(\%):$ C 63.63; H 5.15; N 5.30. Found: C 63.74; H 5.20; N 5.19.

3.4 .12

2-[(2-Butyl-1,3-dioxo-2,3-dihydro-1H-benzo[de]isoquinolin-6-yl)(2-hydroxyethyl)amino]ethyl -2,4-dichlorobenzoate (4f)

Yield 62.3\%, yellow solid, mp 178-179 ${ }^{\circ}$; IR (KBr, $\left.y^{\prime \prime} / \mathrm{cm}^{-1}\right)$ 3377(O-H), 2959-2929 (C-H), 1725-1640 (C=O). ${ }^{1} \mathrm{H}$ NMR $\left(300 \mathrm{MHz}, \mathrm{CDCl}_{3}\right) \delta(\mathrm{ppm}) 8.63-6.76(\mathrm{~m}, 8 \mathrm{H}), 4.83-4.79(\mathrm{t}, J=4.5 \mathrm{~Hz}$, $2 \mathrm{H}), 4.20-4.15(\mathrm{t}, J=7.5 \mathrm{~Hz}, 2 \mathrm{H}), 3.85-3.82(\mathrm{t}, J=4.8 \mathrm{~Hz}, 2 \mathrm{H}), 2.07-2.06(\mathrm{~s}, 1 \mathrm{H}), 1.73-1.72(\mathrm{~m}, 2 \mathrm{H})$, 1.49-1.42 (m, 2H), 1.27(s, 3H), 1.00-0.96 (t, J=4.5 Hz, 3H), $0.86(\mathrm{~m}, 1 \mathrm{H}) .{ }^{13} \mathrm{C}$ NMR $(75 \mathrm{MHz}$, $\left.\mathrm{CDCl}_{3}\right) \delta(\mathrm{ppm}) 165.78,164.61,164.11,148.78,139.14,134.94,134.18,132.77,131.24,131.22$, $129.73,127.56,127.31,125.91,125.14,123.32,120.47,111.42,104.25,63.66,43.42,40.04,40.40$, $30.32,29.72,20.45,13.90$. Anal. Calcd. for $\mathrm{C}_{27} \mathrm{H}_{26} \mathrm{Cl}_{2} \mathrm{~N}_{2} \mathrm{O}_{5}$ (\%): C 61.25; $\mathrm{H} 4.95 ; \mathrm{N}$ 5.29. Found: $\mathrm{C}$ 61.28; H 4.90; N 5.25 .

\section{Conclusion}

A series of novel $N$-n-butyl-1,8-naphthalimide derivatives with mono- and di-substitution at position 4 were synthesized via direct arylation. All of the compounds were characterized by ${ }^{1} \mathrm{H}$ NMR, ${ }^{13} \mathrm{C}$ NMR and MS. To further confirm the structure of the synthesized products, the single-crystal structure of $\mathbf{3 f}$ was determined. The absorption and fluorescent emission of the compounds were investigated. Some electron-donating groups resulted in a blue shift of the emission maxima. The mono-substituted compounds affected the fluorescence intensity of the novel 1,8-naphthalimide derivatives. Among the derivatives, compound 4f exhibited the highest fluorescence intensity with the highest emission maximum. The di-substituent of the hydroxyl group, which exerted a space steric effect, resulted in a bathochromic shift in the emission maximum. Because of this space steric effect, the fluorescence emissions of all of the mono-substituted compounds exceeded those of the di-substituted compounds.

Acknowledgments: This work was supported by the Research Science Foundation in Technology Innovation of Harbin (2015RAYXJ010).

Author contributions: Y. Fu and F. Ye developed the concept of the work. Z.Q. Wang and G. Y. Li carried out the synthetic work. X.X. Pang conducted the fluorescence properties assay. K. Wang contributed to the single crystal culture and discussion. Y. Fu wrote the paper.

Conflict of interest: The authors have no conflicts of interest to declare.

\section{References}


1. Zhang, J.F.; Park, M.; Ren, W.X.; Kim, Y.; Kim, S.J.; Jung, J.H.; Kim, J.S. A pellet-type optical nanomaterial of silica-based naphthalimide-DPA-Cu(II) Complexes: recyclable fluorescence detection of pyrophosphate. Chem. Commun. 2011, 47, 3568-3570. doi:10.1039/c1cc00021g.

2. Gao, C.; Jin, X.; Yan, X.; An, P.; Zhang, Y.; Liu, L.; Tian, H.; Liu, W.; Yao, X.; Tang, Y. A small molecular fluorescent sensor for highly selectivity of zinc ion. Sens. Actuators B Chem. 2013, 176, 775-781. doi:10.1016/j.snb.2012.09.052.

3. Tavallali, H.; Deilamy-Rad, G.; Parhami, A.; Hasanli, N. A novel cyanide-selective colorimetric and fluorescent chemosensor: first molecular security keypad lock based on phosphotungstic acid and CN- inputs. J. Hazard. Mater. 2014, 266, 189-197. doi: 10.1016/j.jhazmat.2013.12.026

4. Nie, H.; Lv, Y.; Yao, L.; Pan, Y.; Zhao, Y.; Li, P.; Sun, G.; Ma, Y.; Zhang, M. Fluorescence detection of trace TNT by novel cross-linking electropolymerized films both in vapor and aqueous medium. J. Hazard. Mater. 2014, 264, 474-480. doi:10.1016/j.jhazmat.2013.09.031.

5. Lee, I.; Oh, W.K.; Jang, J. Screen-printed fluorescent sensors for rapid and sensitive anthrax biomarker detection. J. Hazard. Mater. 2013, 252-253, 186-191. doi:10.1016/j.jhazmat.2013.03.003.

6. Staneva, D.; Grabchev, I.; Betcheva, R. Sensor potential of 1,8-naphthalimide and its dyeing ability of cotton fabric. Dyes Pigm. 2013, 98, 64-70. doi:10.1016/j.dyepig.2013.01.019.

7. Malkondu, S.; Erdemir, S. A novel perylene-bisimide dye as "turn on" fluorescent sensor for $\mathrm{Hg}^{2+}$ ion found in DMF/H2O. Dyes Pigm. 2015, 113, 763-769. doi:10.1016/j.dyepig.2014.10.020.

8. Mutoh, K.; Shima, K.; Yamaguchi, T.; Kobayashi, M.; Abe, J. Photochromism of a naphthalene-bridged imidazole dimer constrained to the "anti" conformation. Org. Lett. 2013, 15, 2938-2941. doi:10.1021/ol401012u.

9. Zhang, Z.; Chen, Y.; Xu, D.; Yang, L.; Liu, A. A new 1, 8-naphthalimide-based colorimetric and “turn-on" fluorescent $\mathrm{Hg}^{2+}$ sensor. Spectrochim. Acta A 2013, 105, 8-13. doi:10.1016/j.saa.2012.11.113.

10. Liu, D.; Qi, J.; Liu, X.; Cui, Z.; Chang, H.; Chen, J.; Yang, G. 4-amino-1,8-naphthalimide-based fluorescent $\mathrm{Cd}^{2+}$ sensor with high selectivity against $\mathrm{Zn}^{2+}$ and its imaging in living cells. Sens. Actuators B Chem. 2014, 204, 655-658. doi:10.1016/j.snb.2014.08.031.

11. Wu, X.; Ma, Q.; Wei, X.; Hou, Y.; Zhu, X. A selective fluorescent sensor for $\mathrm{Hg}^{2+}$ based on covalently immobilized naphthalimide derivative. Sens. Actuators B Chem. 2013, 183, 565-573. doi:10.1016/j.snb.2013.04.024.

12. Adewuyi, S.; Ondigo, D.A.; Zugle, R.; Tshentu, Z.; Nyokong, T.; Torto, N. A highly selective and sensitive pyridylazo-2-naphthol-poly (acrylic acid) functionalized electrospun nanofiber fluorescence "turn-off" chemosensory system for $\mathrm{Ni}^{2+}$. Anal. Methods 2012, 4, 1729-1735. doi:10.1039/c2ay25182e.

13. Azadbakht, R.; Khanabadi, J. A highly sensitive and selective off-on fluorescent chemosensor for $\mathrm{Al}^{3+}$ Based on naphthalene derivative. Inorg. Chem. Commun. 2013, 30, 21-25. doi:10.1016/j.inoche.2013.01.009.

14. Duan, L.; $\mathrm{Xu}, \mathrm{Y}$.; Qian, $\mathrm{X}$. Highly sensitive and selective $\mathrm{Pd}^{2+}$ sensor of naphthalimide derivative based on complexation with alkynes and thio-heterocycle. Chem. Commun. (Camb.) 2008, 47, 6339-6341. doi:10.1039/b815298e.

15. Li, Z.; Zhou, Y.; Yin, K.; Yu, Z.; Li, Y.; Ren, J. A new fluorescence "turn-on" type chemosensor for $\mathrm{Fe}^{3+}$ based on naphthalimide and coumarin. Dyes Pigments 2014, 105, 7-11. doi:10.1016/j.dyepig.2013.12.032.

16. Alaei, P.; Rouhani, S.; Gharanjig, K.; Ghasemi, J. A new polymerizable fluorescent PET chemosensor of fluoride $(\mathrm{F}-)$ based on naphthalimide-thiourea dye. Spectrochim. Acta A Mol. Biomol. Spectrosc. 2012, 90, 85-92. doi:10.1016/j.saa.2012.01.008.

17. Ren, J.; Wu, Z.; Zhou, Y.; Li, Y.; Xu, Z. Colorimetric fluoride sensor based on 1,8-naphthalimide derivatives. Dyes Pigm. 2011, 91, 442-445. doi:10.1016/j.dyepig.2011.04.012.

18. Georgiev, N.I.; Bojinov, V.B.; Marinova, N. Novel PAMAM Light-harvesting antennae based on 1,8-naphthalimide: synthesis, energy transfer, photophysical and $\mathrm{pH}$ sensing properties. Sens. Actuators B Chem. 2010, 150, 655-666. doi:10.1016/j.snb.2010.08.023. 
19. Umeda, R.; Nishida, H.; Otono, M.; Nishiyama, Y. $\pi$-sufficient heteroaromatic compounds fused naphthalimide unit as novel solvatochromic fluorophores. Tetrahedron Lett. 2011, 52, 5494-5496. doi:10.1016/j.tetlet.2011.08.066.

20. Singh, N.; Kaur, N.; McCaughan, B.; Callan, J.F. Ratiometric fluorescent detection of Cu (II) in semi-aqueous solution using a two-fluorophore approach. Tetrahedron Lett. 2010, 51, 3385-3387. doi:10.1016/j.tetlet.2010.04.099.

21. I. Ott, X. Qian, Y. Xu, D.H. Vlecken, I.J. Marques, D. Kubutat, J. Will, W.S. Sheldrick, P. Jesse, A. Prokop, C.P. Bagowski, A gold (I) phosphine complex containing a naphthalimide ligand functions as a TrxR inhibiting antiproliferative agent and angiogenesis inhibitor. J. Med. Chem. 2009, 52, 763-770. doi:10.1021/jm8012135.

22. Yang, J.; Wang, X.; Xu, L. Studies on the synthesis and spectral properties of novel 4-benzofuranyl-1,8-naphthalimide derivatives. Dyes Pigm. 2005, 67, 27-33. doi:10.1016/j.dyepig.2004.09.017.

23. Jin, Z.N.; Li, N.J.; Wang, C.F.; Jiang, H.J.; Lu, J.M.; Zhou, Q.Z. Synthesis and fluorescence property of some novel 1,8-naphthalimide derivatives containing a thiophene ring at the C-4 position. Dyes Pigm. 2013, 96, 204-210. doi:10.1016/j.dyepig.2012.07.018.

24. Sheldrick, G.M., SHELXTL Version 5.1, Bruker Analytical X-ray Instruments Inc. Madison Book Company: WI, 1998.

25. Sheldrick, G.M., SHELXL-97, PC Version. University of Göttingen: Germany, 1997.

26. Liu, B.; Tian, H. A selective fluorescent ratiometric chemodosimeter for mercury ion. Chem. Commun. 2005, 25, 3156-3158. doi:10.1039/b501913c.

27. Guo, X.F.; Zhu, B.C.; Liu, Y.Y.; Zhang, Y.; Jia, L.H.; Qian, X.H. Synthesis and properties of N-Butyl-4-(aza-15-crown-5)-1,8-naphthalimide as a fluorescent robe. Chin. J. Org. Chem. 2006, 26, 504-507.

28. Wang, H.; Yang, L.; Zhang, W.; Zhou, Y.; Zhao, B.; Li, X. A colorimetric probe for copper (II) Ion based on 4-amino-1,8-naphthalimide. Inorg. Chim. Acta 2012, 381, 111-116. doi:10.1016/j.ica.2011.07.039.

29. Lee, S.; Lee, J.H.; Pradhan, T.; Lim, C.S.; Cho, B.R.; Bhuniya, S.; Kim, S.; Kim, J.S. Fluorescent turn-on $\mathrm{Zn}^{2+}$ sensing in aqueous and cellular media. Sens. Actuators B Chem. 2011, 160, 1489-1493. doi:10.1016/j.snb.2011.07.065.

30. Jiang, W.; Sun, Y.; Wang, X.; Wang, Q.; Xu, W. Synthesis and photochemical properties of novel 4-diarylamine-1,8-naphthalimide derivatives. Dyes Pigm. 2008, 77, 125-128. doi:10.1016/j.dyepig.2007.03.017.

31. Bardajee, G.R. Microwave-assisted solvent-free synthesis of fluorescent naphthalimide dyes. Dyes Pigm. 2013, 99, 52-58. doi:10.1016/j.dyepig.2013.04.004.

32. Yang, J.X.; Wang, X.; Wang, X.; Xu, L. The synthesis and spectral properties of novel 4-phenylacetylene-1,8-naphthalimide derivatives. Dyes Pigm. 2005, 66, 83-87. doi:10.1016/j.dyepig.2004.07.015. 